\title{
Screening key miRNAs and genes in prostate cancer by microarray analysis
}

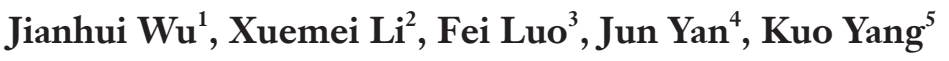 \\ ${ }^{1}$ Department of Urology, Tianjin First Central Hospital, Tianjin 300192, China; ${ }^{2}$ Department of Endocrinology, Key Laboratory of Hormones \\ and Development (Ministry of Health), Tianjin Key Laboratory of Metabolic Diseases, Tianjin Metabolic Diseases Hospital \& Tianjin Institute \\ of Endocrinology, Tianjin Medical University, Tianjin 300070, China; ${ }^{3}$ Department of Urology, Tianjin Union Medical Center, Tianjin 300121, \\ China; ${ }^{4}$ Department of Pathology, Tianjin First Central Hospital, Tianjin 300192, China; ${ }^{5}$ Department of Urology, Tianjin Institute of Urology, The \\ Second Hospital of Tianjin Medical University, Tianjin 300211, China \\ Contributions: (I) Conception and design: J Wu, K Yang; (II) Administrative support: K Yang; (III) Provision of study materials or patients: J Wu, \\ X Li, F Luo, J Yan; (IV) Collection and assembly of data: J Wu, X Li, F Luo, J Yan; (V) Data analysis and interpretation: J Wu, X Li, F Luo; (VI) \\ Manuscript writing: All authors; (VII) Final approval of manuscript: All authors. \\ Correspondence to: Kuo Yang. Department of Urology, Tianjin Institute of Urology, The Second Hospital of Tianjin Medical University. No. 23, \\ Pingjiang Road, Hexi District, Tianjin 300211, China. Email: kuoyang01@163.com.
}

Background: Prostate cancer (PCa) is the second most frequent cancer and the fifth leading cause of cancer-related death in men while the mechanisms remain unclear.

Methods: Differentially expressed mRNAs (DEmRNAs) and miRNAs (DEmiRNAs) between PCa and non-tumor controls were identified by using microarray analysis. Functional annotation of DEmRNAs, construction of protein-protein interaction (PPI) network and prediction of upstream transcription factors and downstream target DEmRNAs of DEmiRNAs were conducted to further research functions of key DEmRNAs and DEmiRNAs. Validation of selected DEmRNAs and survival analysis were conducted by using The Cancer Genome Atlas (TCGA).

Results: Total of 91 DEmRNAs and 62 DEmiRNAs were obtained. Thrombospondin-4 precursor (THBS4) was the most significantly up-regulated DEmRNA whose product was predicted to interact with the hub protein of the PCa-specific PPI network, collagen type I alpha 1 chain (COL1A1). Both ATP binding cassette subfamily $\mathrm{C}$ member 4 (ABCC4) and endothelin receptor type $\mathrm{B}$ (EDNRB) have great prognostic value for PCa. Thrombospondin type 1 domain containing 4 (THSD4) was a down-regulated DEmRNA regulated by several cancer-related miRNAs including has-miR-107, hsa-miR-3175 and hsamiR-484. Two miRNAs (hsa-miR-428 and hsa-miR-4284) involve in PCa by regulating BMP5-BAMBI interaction and TGF-beta signaling pathway. The expression of selected DEmRNAs between PCa and nontumor controls in TCGA was consistent with that in our microarray analysis, generally.

Conclusions: Key DEmRNAs and DEmiRNAs between PCa and non-tumor controls were identified in this study which provided clues for exploring the molecular mechanism and developing potential biomarkers and therapeutic target sites for PCa.

Keywords: Prostate cancer (PCa); microarray analysis; differentially expressed genes; miRNA

Submitted Jul 30, 2019. Accepted for publication Nov 29, 2019.

doi: $10.21037 /$ tcr.2019.12.30

View this article at: http://dx.doi.org/10.21037/tcr.2019.12.30 


\section{Introduction}

Prostate cancer $(\mathrm{PCa})$ is the second most frequent cancer and the fifth leading cause of cancer-related death in men (1). It is estimated that there will be 1.7 million new cases annually worldwide by 2030 (2). However, the mechanisms underlying the development and progression of $\mathrm{PCa}$ remain to be fully elucidated. Hence, there is an urgent needed for exploring the molecular pathogenesis and developing novel biomarkers and therapeutic strategies for PCa.

$\mathrm{PCa}$ carcinogenesis have been reported to be associated with multiple alterations in oncogenes and tumor suppressor genes (3-5). MicroRNAs (miRNAs) are short non-coding RNAs ( 22 nucleotides) that regulate up to $30 \%$ of human genes post-transcriptionally which represses translation or degrades messenger RNA (mRNA) and play a crucial role in multiple cellular processes such as proliferation, differentiation, apoptosis, migration, and invasion $(6,7)$. Furthermore, accumulated evidence indicated that miRNA play important roles in the processes of PCa (8-10).

In this present study, differentially expressed mRNAs (DEmRNAs) and miRNAs (DEmiRNAs) were identified between PCa and non-tumor tissues by microarray analysis. Functional annotation of DEmRNAs, construction of protein-protein interaction (PPI) network and prediction of upstream transcription factors and downstream target DEmRNAs of DEmiRNAs were conducted to further research functions of key DEmRNAs and DEmiRNAs. Our study may provide new clues for exploring molecular mechanism of PCa and developing PCa-associated diagnostic and therapeutic approaches.

\section{Methods}

\section{Patients}

Seven patients with PCa and two patients with benign prostatic hyperplasia (BPH) were recruited in this study from The 2nd Hospital of Tianjin medical university between June 2010 and June 2011. The inclusion criteria were as follows: (I) patients with PCa (T2N0M0) were diagnosed based on pathological examination and MRI results; (II) age and sex matched patients with $\mathrm{BPH}$ were diagnosed via biopsy. Table 1 displayed the detailed information of all these participants.

This study has been approved by the ethics institute of The 2 nd Hospital of Tianjin medical university (KY2014K016). All these participants signed the informed consent. This research complied with the principles of the
Declaration of Helsinki (as revised in 2013).

\section{Preparation of RNA}

Pca tumor tissue samples (PCa_1-PCa_7) were obtained from the seven patients with $\mathrm{PCa}$ (PCa1-PCa7). Five adjacent non-tumor tissue samples (Con_1-Con_5) obtained from five patients with $\mathrm{PCa}$ (PCa1-PCa5) and two prostate tissue samples (Con_6-Con_7) obtained from two patients with $\mathrm{BPH}(\mathrm{BPH} 1-\mathrm{BPH} 2)$ were serve as non-tumor controls. According to the manufacturer's instructions, total RNA from each sample was isolated and purified by using Trizol (Invitrogen, Gaithersburg, MD, USA) and NucleoSpin ${ }^{\circledR}$ RNA clean-up (MACHEREY-NAGEL, Germany), respectively. The concentration and purity of the total RNA were assayed with a Nanodrop spectrophotometer (Thermo Fisher Scientifc Inc., Waltham, MA, USA). The integrity of the total RNA was confirmed using electrophoresis in agarose gel containing formaldehyde. By using MirVana ${ }^{\circledR}$ miRNA isolation Kit (Ambion, AM1560), miRNA was isolated from each sample.

\section{Gene array profiling}

By using Jingxin cRNA linear amplification and labeling kit (CapitalBio) (11), RNA from each sample was labeled with Cy3-dCTP or Cy5-Dctp. Then, labeled products were purified with the PCR NucleoSpin Extract II kit (MacheryNagel Inc.) and hybridized with the 35k human Genome Array (CapitalBio Inc., Beijing, China). After hybridization, the gene arrays were washed and scanned with CapitalBio LuxScan $^{\mathrm{TM}} 10 \mathrm{~K}-\mathrm{A}$ scanners. LuxScan 3.0, BoaoAnalyzer6_ step1.pl and BoaoAnalyzer6_step2.pl software products (CapitalBio Inc.) were used to extract and analyze the data. The fluorescence intensity was normalized prior to analysis.

\section{MiRNA array profiling}

By using the FlashTagTM Biotin RNA Labeling Kit (Genisphere, LLC, Hatfeld, PA, USA), miRNA was biotin labeled according to the manufacturer's instructions. Then, biotin labeled miRNAs was hybridized to Affymetrix ${ }^{\circledR}$ GeneChip ${ }^{\circledR}$ miRNA Arrays (Affymetrix, Santa Clara, CA, USA) with the Eukaryotic Hybridization Control Kit (Affymetrix, P/N 900454) in the Hybridization Oven 640 (Affymetrix). Hybridized miRNA arrays were washed and stained using the Hybridization, Wash and Stain Kit (Affymetrix) on the Fluidics Station 450 (Affymetrix) and 
Table 1 Patient characteristic

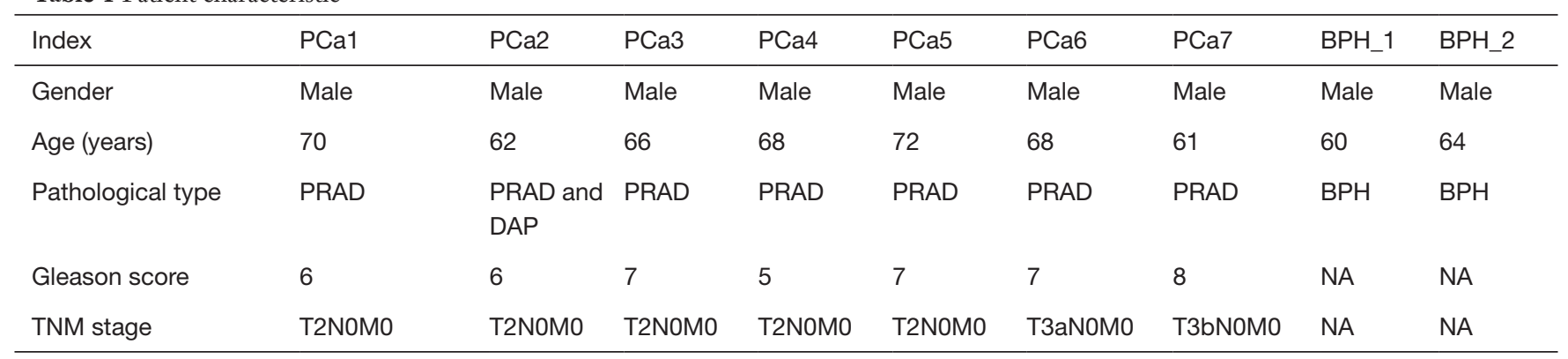

$\mathrm{PCa}$, prostate cancer; PRAD, prostate adenocarcinoma; DAP, ductal adenocarcinoma of the prostate.

scanned on a GeneChip ${ }^{\circledR}$ Scanner 3000 (Affymetrix) using Command Console ${ }^{\mathrm{TM}}$ 1. Data summarization and quality control were performed by using Affymetrix miRNA QC tool. With the Robust Multichip Average (RMA), background correction and normalization were conducted.

\section{Identification of DEmRNAs and DEmiRNAs}

The gene array data and the miRNA array data obtained were normalized by locally weighted scatterplot smoothing (LOWESS) method (12). Then, the $t$-test method in the LIMMA package of $\mathrm{R}$ software (13) was used to identify the DEmRNAs and DEmiRNAs between PCa and non-tumor controls. $\mid \log 2$ Foldchange $\mid>1$ and $\mathrm{P}$ value $<0.05$ were the thresholds for the DEmRNAs and $\mathrm{P}$ value $<0.05$ was the threshold for DEmiRNAs. Hierarchical clustering analysis of DEmiRNAs and DEmRNAs were conducted by using $\mathrm{R}$ package "pheatmap".

\section{PPI network}

To further research the biological functions of DEmRNAs between PCa and non-tumor controls, the PPI network was constructed by using STRING (http://string-db.org/ cgi/about.pl?sessionId=DfAjFN3R1SaY\&footer_active_ subpage=content) and the Cytoscape software. Nodes and edges were used to represent the proteins and interactions between two proteins, respectively. Proteins with a degree of $\geq 5$ were defined as hub proteins of this PPI network.

\section{Functional annotation}

Gene ontology (GO) and Kyoto Encyclopedia of Genes and Genomes (KEGG) pathway enrichment analysis of DEmRNAs between PCa and non-tumor controls were conducted by using online-based software DAVID (https:// david.ncifcrf.gov/). Statistical significance was defined as $\mathrm{P}$ value $<0.05$.

\section{Prediction of potential transcription factors of DE-miRNAs}

FunRich software, is a tool mainly used for functional enrichment and interaction network analysis of genes and proteins (14). By using FunRich software, the upstream transcription factors of DEmiRNAs were predicted.

\section{DEmiRNA-DEmRNA interaction analysis}

Four bioinformatic algorithms (miRDB, TarPmiR, miRTarBase and Targetscan) with miRwalk 3.0 (http:// miRwalk.umm.uni-heidelberg.de/) were used to predict the downstream target genes. Then, DEmiRNA-DEmRNA pairs predicted by these four bioinformatic algorithms in which DEmRNA was negatively correlated with DEmiRNAs in this study were retained for our following research. Based on these above DEmiRNA-DEmRNA pairs, the DEmiRNA-DEmRNA interaction network was constructed by the Cytoscape software.

\section{Validation for the expression of DEmRNAs}

The Cancer Genome Atlas (TCGA; https://tcga-data.nci. nih.gov/tcga/) is a public-funded project which consists of multi-dimensional data of for multiple cancers at DNA, RNA, and protein levels. The mRNA profile of 492 prostate adenocarcinoma (PRAD) cases and 52 adjacent non-tumor controls was stored in TCGA. Gene Expression Profiling Interactive Analysis (GEPIA, http://gepia.cancerpku.cn/) is a web-based tool to deliver fast and customizable functionalities based on TCGA and GTEx data (15). By 
using GEPIA, expression of selected DEmRNAs obtained in this study were validated by TCGA.

\section{Survival analysis}

To evaluate the prognostic value of all these obtained DEmRNAs in PCa, survival analysis was performed for all these DEmRNAs by using GEPIA.

\section{Results}

DEmRNAs and DEmiRNAs between PCa and non-tumor controls

Total of 91 DEmRNAs (51 up-regulated and 40 downregulated DEmRNAs) and 62 DEmiRNAs (39 upregulated and 23 down-regulated DEmiRNAs) between $\mathrm{PCa}$ and non-tumor controls were obtained. Top 20 significantly DEmRNAs and DEmiRNAs were displayed in Tables 2 and 3, respectively. Hierarchical cluster analysis of DEmRNAs and DEmiRNAs were shown in Figure $1 A$ and $B$, respectively.

\section{Functional annotation}

The most significantly enriched GO terms in PCa were extracellular space $(\mathrm{P}=5.71 \mathrm{E}-05)$, extracellular exosome $(\mathrm{P}=3.44 \mathrm{E}-04)$, cell adhesion $(\mathrm{P}=4.65 \mathrm{E}-04)$, extracellular matrix $(\mathrm{P}=1.11 \mathrm{E}-03)$ and membrane raft $(\mathrm{P}=1.33 \mathrm{E}-03)$. The significantly enriched GO terms including "biological process", "molecular function", and "cellular component" were displayed in Figure $2 A$. TGF-beta signaling pathway $(\mathrm{P}=5.62 \mathrm{E}-03)$ was the only pathway that enriched for DEmRNAs between PCa and non-tumor controls. Four DEmRNAs including inhibin subunit beta A (INHBA) and BMP and activin membrane bound inhibitor (BAMBI), inhibitor of DNA binding 4, HLH protein (ID4) and bone morphogenetic protein 5 (BMP5) were enriched in this pathway (Figure 2B).

\section{Prediction of upstream transcription factors of DEmiRNAs}

After analysis by using FunRich software, upstream transcription factors of DEmiRNAs were predicted. The top 10 transcription factors for DEmiRNAs (Figure $3 A$ ) were early growth response 1 (EGR1), POU class 2 homeobox 1 (POU2F1), NK6 homeobox 1 (NKX6-1), Sp1 transcription factor (SP1), NOBOX oogenesis homeobox (NOBOX), AT-rich interaction domain 3A (ARID3A),
YY1 transcription factor (YY1), homeobox A9 (HOXA9), myogenic factor 5 (MYF5), POU class 4 homeobox 3 (POU4F3).

\section{DEmiRNAs-DEmRNAs interaction network}

A total of 54 DEmiRNA-DEmRNA pairs consisted of 24 DEmiRNAs (12 up-regulated miRNAs and 2 downregulated mRNAs) and 14 DEmRNAs (5 up-regulated mRNAs and 19 down-regulated mRNAs) were obtained. The DEmiRNA-DEmRNA interaction network was displayed in Figure 3B, hsa-miR-484, hsa-miR-1231 and hsa-miR-941 were three hub miRNAs.

\section{PPI network}

The PPI network of DEmRNAs were consisted of 36 nodes and 30 edges (Figure 4). Two hub proteins including collagen type I alpha 1 chain $($ COL1A1, degree $=7$ ) and Thy- 1 cell surface antigen (THY1, degree $=5$ ) were identified based on this PPI network (Figure 4).

\section{Validation of selected DEmRNAs in TCGA}

Based on our microarray analysis, three up-regulated DEmRNAs including thrombospondin-4 precursor (THBS4), PDZ and LIM domain protein 5 (PDLIM5) and ATP binding cassette subfamily C member 4 (ABCC4) and five down-regulated DEmRNAs including bone morphogenetic protein 5 precursor (BMP5), FXYD domain-containing ion transport regulator 1 (FXYD1), inhibitor of DNA binding 4 (ID4), thrombospondin type 1 domain containing 4 (THSD4) and endothelin receptor type B (EDNRB) were selected to perform the expression validation by TCGA. The different expression levels of these eight DEmRNAs between PRAD and adjacent nontumor controls in TCGA were analyzed and depicted through box-plots (Figure 5) which were consistent with our microarray analysis, generally.

\section{Survival analysis}

Up-regulation of ABCC4 is significantly associated with poor survival of patients with $\mathrm{PCa}(\mathrm{P}=0.045$, Figure $6 A)$. Decreased EDNRB predicts poor survival in $\mathrm{PCa}(\mathrm{P}=0.027$, Figure $6 B$ ). Both these two genes have great prognostic value for PCa.

Discussion 
Table 2 Top 20 up-regulated and down-regulated DEmRNAs between $\mathrm{PCa}$ and non-tumor controls

\begin{tabular}{|c|c|c|c|}
\hline Gene ID & Gene symbol & Log2FC & $P$ value \\
\hline ENSG00000113296 & THBS4 & 3.420962 & 0.030016 \\
\hline ENSG00000198542 & ITGBL1 & 3.116457 & 0.036118 \\
\hline ENSG00000169507 & NP_775783.1 & 3.067172 & 0.049361 \\
\hline ENSG00000144355 & $D L X 1$ & 2.998112 & 0.044163 \\
\hline ENSG00000105664 & COMP & 2.532081 & 0.047278 \\
\hline ENSG00000106819 & $A S P N$ & 2.481949 & 0.025225 \\
\hline ENSG00000132821 & C20orf102 & 2.271759 & 0.006654 \\
\hline ENSG00000153982 & GDPD1 & 2.24658 & 0.025275 \\
\hline ENSG00000109625 & $C P Z$ & 2.028859 & 0.031082 \\
\hline ENSG00000147676 & MAL2 & 1.979245 & 0.047306 \\
\hline ENSG00000170369 & CST2 & 1.893333 & 0.027599 \\
\hline ENSG00000164687 & FABP5 & 1.891178 & 0.044616 \\
\hline ENSG00000163110 & PDLIM5 & 1.807621 & 0.02019 \\
\hline ENSG00000154917 & $R A B 6 B$ & 1.771981 & 0.029096 \\
\hline ENSG00000187210 & GCNT1 & 1.756058 & 0.009711 \\
\hline ENSG00000177707 & PVRL3 & 1.654416 & 0.020207 \\
\hline ENSG00000105707 & $H P N$ & 1.653384 & 0.00697 \\
\hline ENSG00000183010 & PYCR1 & 1.647304 & 0.011148 \\
\hline ENSG00000140479 & PCSK6 & 1.612633 & 0.02487 \\
\hline ENSG00000122641 & INHBA & 1.59196 & 0.025026 \\
\hline ENSG00000074590 & NUAK1 & -3.677414 & 0.033604 \\
\hline ENSG00000112175 & BMP5 & -2.569483 & 0.031053 \\
\hline ENSG00000143196 & $D P T$ & -2.213348 & 0.018534 \\
\hline ENSG00000172005 & $M A L$ & -2.184924 & 0.034631 \\
\hline ENSG00000101938 & CHRDL1 & -2.024137 & 0.044497 \\
\hline ENSG00000186847 & $K R T 14$ & -1.955376 & 0.048046 \\
\hline ENSG00000049319 & SRD5A2 & -1.933853 & 0.041699 \\
\hline ENSG00000096141 & LY6G6D & -1.920051 & 0.048276 \\
\hline ENSG00000110244 & APOA4 & -1.89642 & 0.046703 \\
\hline ENSG00000204789 & ZNF204 & -1.877634 & 0.016781 \\
\hline ENSG00000154188 & ANGPT1 & -1.7652 & 0.036458 \\
\hline ENSG00000126258 & FXYD1 & -1.747271 & 0.03719 \\
\hline ENSG00000162641 & C1orf62 & -1.721416 & 0.008241 \\
\hline ENSG00000151778 & C13orf21 & -1.703242 & 0.041983 \\
\hline ENSG00000165646 & SLC18A2 & -1.686371 & 0.047647 \\
\hline ENSG00000176049 & JAKMIP2 & -1.471425 & 0.025972 \\
\hline
\end{tabular}

Table 2 (continued)
Table 2 (continued)

\begin{tabular}{llll}
\hline Gene ID & Gene symbol & Log2FC & P value \\
\hline ENSG00000137273 & FOXF2 & -1.463853 & 0.035233 \\
ENSG00000161055 & SCGB3A1 & -1.463547 & 0.00583 \\
ENSG00000173376 & C4orf31 & -1.446799 & 0.046379 \\
ENSG00000172201 & ID4 & -1.420409 & 0.020618 \\
\hline
\end{tabular}

$\mathrm{PCa}$, prostate cancer; DEmRNAs, differentially expressed mRNAs; Log2FC, log2Fold change.

PCa is the most lethal urogenital system malignancy in men. Therefore, investigating the molecular mechanisms involved in the progression of PCa is crucial and may reveal novel biomarkers and therapeutic strategies. In this study, DEmRNAs and DEmiRNAs between PCa and non-tumor controls were identified by microarray analysis and their potential roles were further explored by bioinformatics analysis.

THBS4 was the most significantly up-regulated gene between PCa and non-tumor controls in this study. As a member of the extracellular calcium-binding protein family (16), THBS4 was reported to involve with various cancers including colorectal, gastric and prostate cancers (17-19). Furthermore, increased THBS4 has been demonstrated to play a significant role in the proliferation and migration of $\mathrm{PCa}$ by regulating the matrix metalloproteinases-9 (MMP-9) and p38 Mitogenactivated protein kinase (MAPK) signaling pathway (4). In this present study, interaction between THBS4 and a potential regulator in metastasis of $\mathrm{PCa}, \mathrm{COL} 1 \mathrm{~A} 1(5,8)$ was found which provided clues for exploring the precise role of THBS4 in PCa.

Two DEmRNAs including ABCC4 and EDNRB with great prognostic value for $\mathrm{PCa}$ were identified in this study which might serve as potential biomarkers. Highly methylated EDNRB $(20,21)$ and dysregulated ABCC4 $(22,23)$ have been reported to be regulators of PCa. In addition, EDNRB could be regulated by both hsa-miR-107 and hsa-miR-762. Hsa-miR-107 was a potential regulator of multiple cancers (24-26). Aberrant expressed hsa-miR-107 has been found in meningioma (25) which promoted the proliferation and inhibited apoptosis of colon cancer cells (26). Therefore, hsa-miR-107/hsa-miR-762-EDNRB interactions might play a key role in the processes of $\mathrm{PCa}$.

According to the KEGG enrichment analysis, TGF- 
Table 3 Top 20 up-regulated and down-regulated DEmiRNAs between PCa and non-tumor controls

\begin{tabular}{|c|c|c|}
\hline miRNA & $\log 2 \mathrm{FC}$ & $P$ value \\
\hline hsa-miR-183 & 1.572899 & 0.047641 \\
\hline hsa-miR-182 & 1.553273 & 0.03888 \\
\hline hsa-miR-4284 & 1.318899 & 0.04523 \\
\hline hsa-miR-663b & 1.283888 & 0.016842 \\
\hline hsa-miR-375 & 1.229287 & 0.021521 \\
\hline hsa-miR-941 & 1.083061 & 0.018677 \\
\hline hsa-miR-3180-3p & 0.926267 & 0.043474 \\
\hline hsa-miR-93 & 0.893455 & 0.015524 \\
\hline hsa-miR-106b & 0.885544 & 0.047635 \\
\hline hsa-miR-1231 & 0.866421 & 0.034641 \\
\hline hsa-miR-217 & 0.823899 & 0.04281 \\
\hline hsa-miR-1292 & 0.742308 & 0.020546 \\
\hline hsa-miR-25 & 0.733483 & 0.015988 \\
\hline hsa-miR-484 & 0.692888 & 0.027458 \\
\hline hsa-miR-1307 & 0.687092 & 0.023083 \\
\hline hsa-miR-339 & 0.653019 & 0.03867 \\
\hline hsa-miR-200c & 0.620878 & 0.013658 \\
\hline hsa-miR-3175 & 0.610865 & 0.023216 \\
\hline hsa-miR-23a & 0.601889 & 0.022816 \\
\hline hsa-miR-1972 & 0.550624 & 0.016358 \\
\hline hsa-miR-376c & -1.10784 & 0.031947 \\
\hline hsa-miR-1 & -1.04235 & 0.046145 \\
\hline hsa-miR-30e & -0.82655 & 0.014027 \\
\hline hsa-miR-221 & -0.81939 & 0.006079 \\
\hline hsa-miR-503 & -0.77411 & 0.025611 \\
\hline hsa-miR-99a & -0.75539 & 0.006023 \\
\hline hsa-miR-338 & -0.74427 & 0.042402 \\
\hline hsa-miR-15a & -0.73052 & 0.041203 \\
\hline hsa-miR-29a & -0.7237 & 0.007211 \\
\hline hsa-miR-455 & -0.7106 & 0.01735 \\
\hline hsa-miR-455 & -0.70809 & 0.033923 \\
\hline hsa-miR-221 & -0.70405 & 0.044713 \\
\hline hsa-miR-27b & -0.63631 & 0.005391 \\
\hline hsa-miR-422a & -0.63095 & 0.021855 \\
\hline hsa-miR-379 & -0.60175 & 0.04474 \\
\hline hsa-miR-376a & -0.59835 & 0.033791 \\
\hline hsa-miR-494 & -0.59444 & 0.011013 \\
\hline hsa-let-7g & -0.5942 & 0.043972 \\
\hline hsa-miR-337 & -0.58311 & 0.039703 \\
\hline hsa-miR-224 & -0.52878 & 0.034879 \\
\hline
\end{tabular}

$\mathrm{PCa}$, prostate cancer; DEmiRNAs, differentially expressed miRNAs; Log2FC, log2 fold change. beta signaling pathway is the sole pathway that enriched for DEmRNAs of PCa. Previous studies have confirmed that TGF-beta signaling pathway is involved in cancer invasion, metastasis and angiogenesis (27). Furthermore, TGFbeta might involve with the regulation of collagen (28), fbronectin (29), laminin (30) and MMP-9 (31) to affect migratory and invasive capacity. BMP5 and BAMBI were two DEmRNAs that enriched in TGF-beta signaling pathway. Aberrant transcriptional expression levels of several bone morphogenetic proteins (BMPs) such as BMP 2-7 have been observed in PCa compared to normal prostate tissues which highlighted the importance of the BMP family in PCa (32). Based on our PPI network, BMP5, a member of BMP family was found to interact with BAMBI, and BAMBI could make an inhibition on BMP as well as TGF-beta signaling pathway (33). Moreover, BMP5 was a shared downstream target of two cancer-related miRNAs, hsa-miR-4284 (34) and hsa-miR-484 $(35,36)$. We speculated that these two miRNAs play potential roles in PCa by regulating BMP5-BAMBI interaction and TGFbeta signaling pathway.

Based on DEmiRNA-DEmRNA interaction network, THSD4 is a down-regulated DEmRNA regulated by a maximum of DEmiRNAs including hsa-miR-107, hsamiR-1972, hsa-miR-3175, hsa-miR-3196, hsa-miR-484, hsa-miR-663b, hsa-miR-762 and hsa-miR-941. Although no previous study reports the association between THSD4 and $\mathrm{PCa}$, THSD 4 has been indicated to involve in tumorigenesis and development of various cancers including breast cancer, glioblastoma and esophageal carcinoma (37-39). Moreover, has-miR-107, hsa-miR-3175 and hsamiR-484 have been proved to be potential mediators of PCa $(26,40,41)$ which suggested that THSD4 might be potential regulators of $\mathrm{PCa}$ with regulation by these $\mathrm{PCa}$-related miRNAs.

\section{Conclusions}

In conclusion, key DEmRNAs and DEmiRNAs between $\mathrm{PCa}$ and non-tumor controls were identified in this study. Both ABCC4 and EDNRB have great prognostic value for PCa which were potential prognostic biomarkers. THSD4 serves as a potential regulator of PCa regulated by several cancer-related miRNAs including has-miR-107, hsa-miR-3175 and hsa-miR-484. Hsa-miR-428 and hsamiR-4284 might play potential roles in PCa by regulating BMP5-BAMBI interaction and TGF-beta signaling pathway. All these findings provided clues for exploring the 


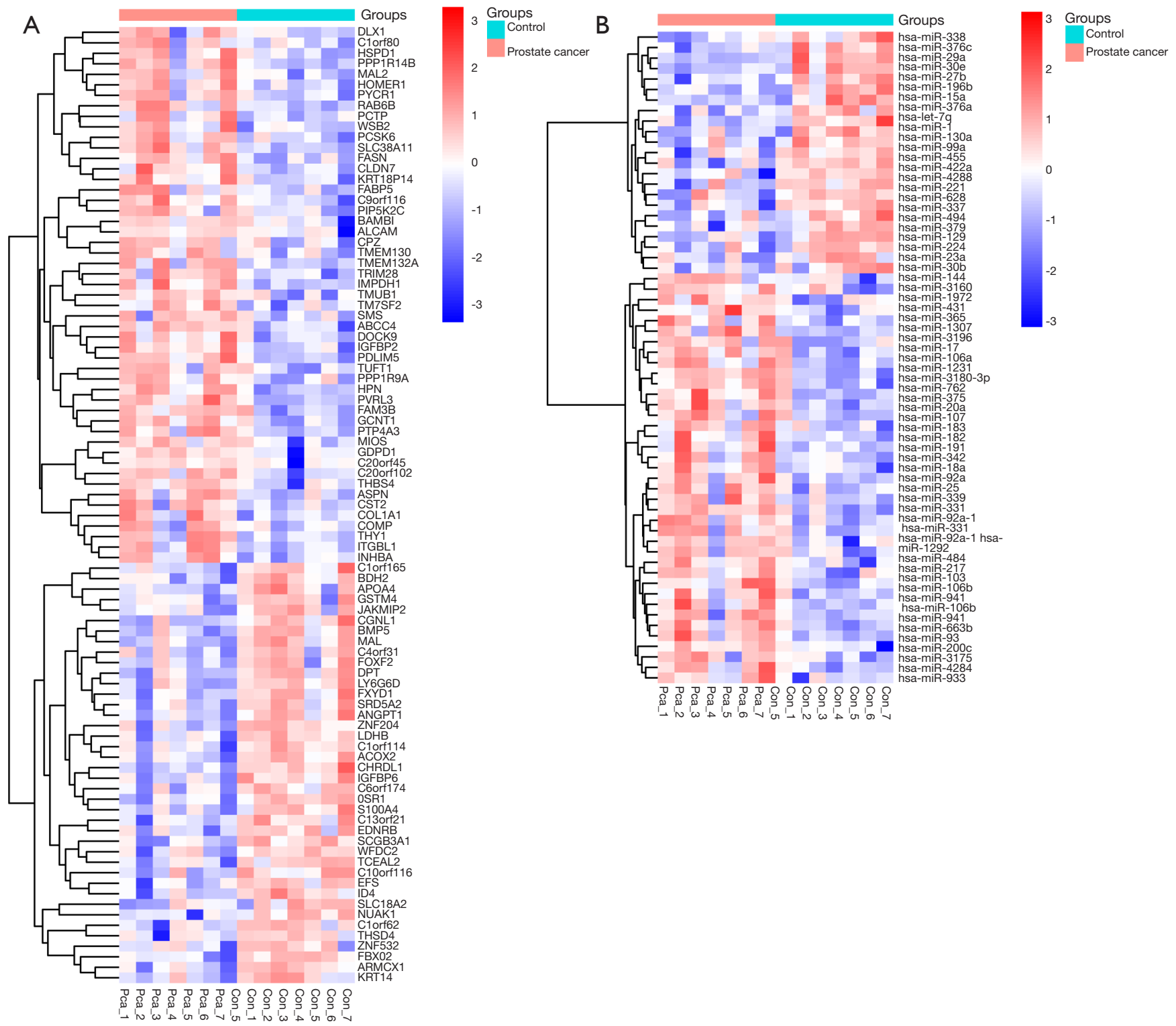

Figure 1 Hierarchical clustering analysis of DEmRNAs and DEmiRNAs between PCa and non-tumor controls. (A) DEmRNAs; (B) DEmiRNAs. Row and column represent DEmRNAs/DEmiRNAs and samples, respectively. The color scale indicated the $\log 2$-transformed expression levels of DEmRNAs and DEmiRNAs. Red and blue color indicate up- and down-regulation, respectively. DEmRNAs, differentially expressed mRNAs; DEmiRNAs, differentially expressed miRNAs; PCa, prostate cancer. 
A

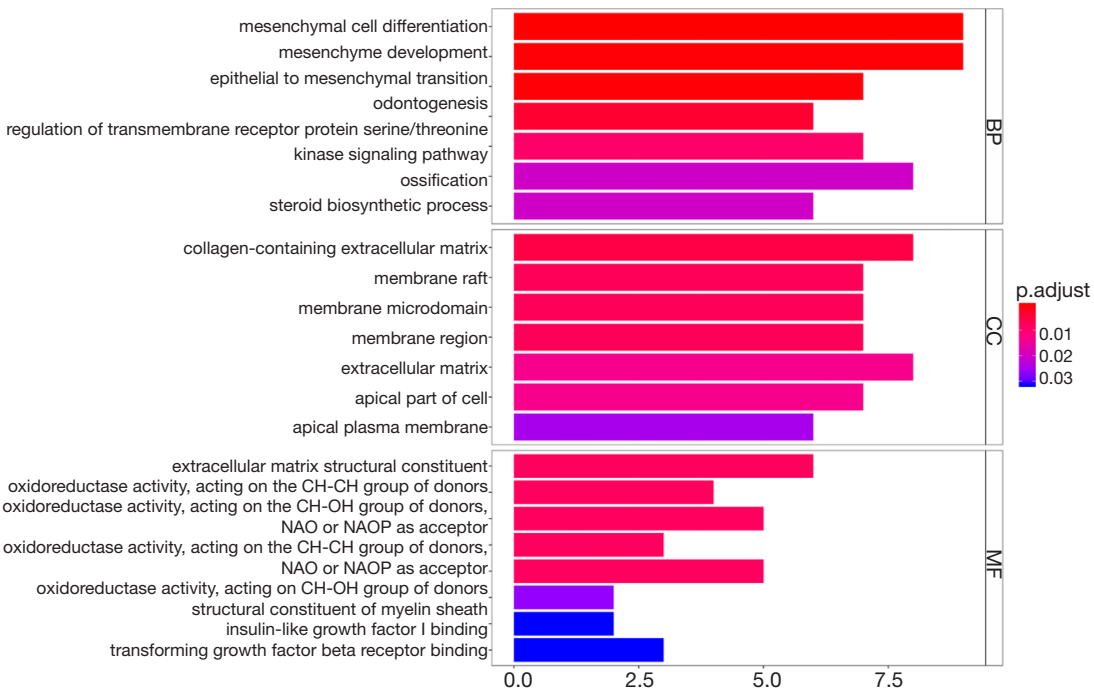

B

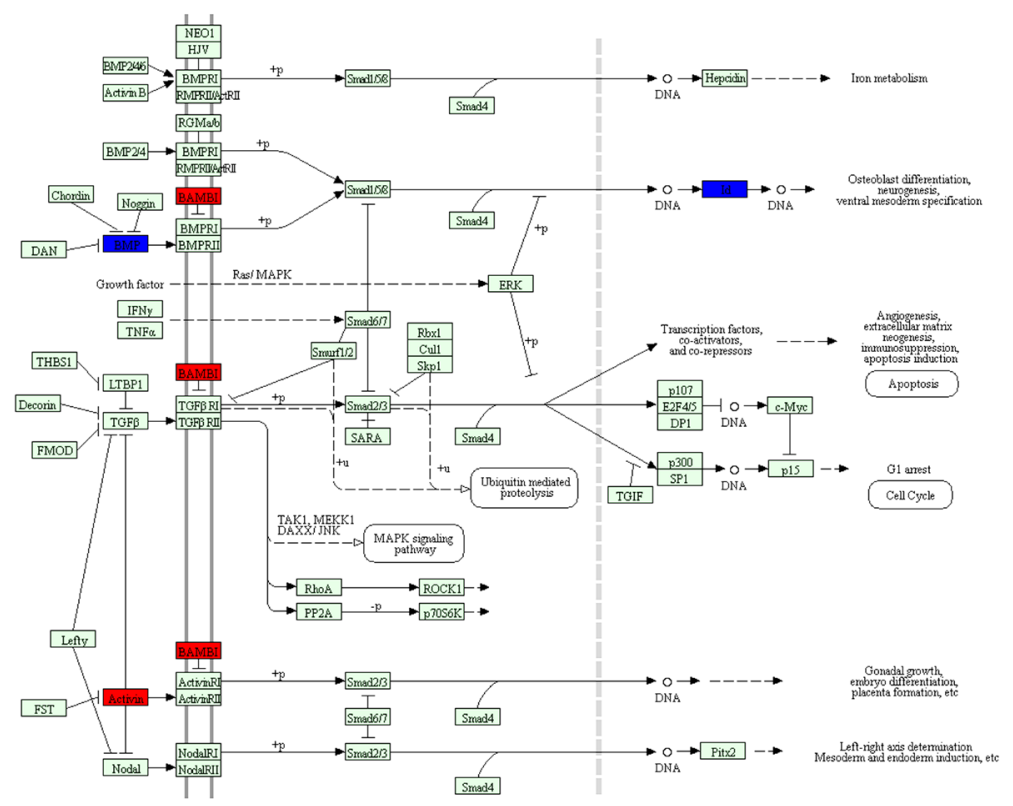

Figure 2 Functional annotation of DEmRNAs. (A) Significantly enriched GO terms including "biological process (BP)", "molecular function (MF)", and "cellular component (CC)" for DEmRNAs between PCa and non-tumor controls. The Y-axis shows GO terms and the $\mathrm{X}$-axis represents counts of DEmRNAs enriched in GO terms. The color scale represents adjust $\mathrm{P}$ value. (B) TGF-beta signaling pathway. The red and blue rectangles represented the particles which regulated by the up- and down-regulated DEmRNAs between PCa and nontumor controls, respectively. DEmRNAs, differentially expressed mRNAs; GO, Gene ontology; PCa, prostate cancer. 


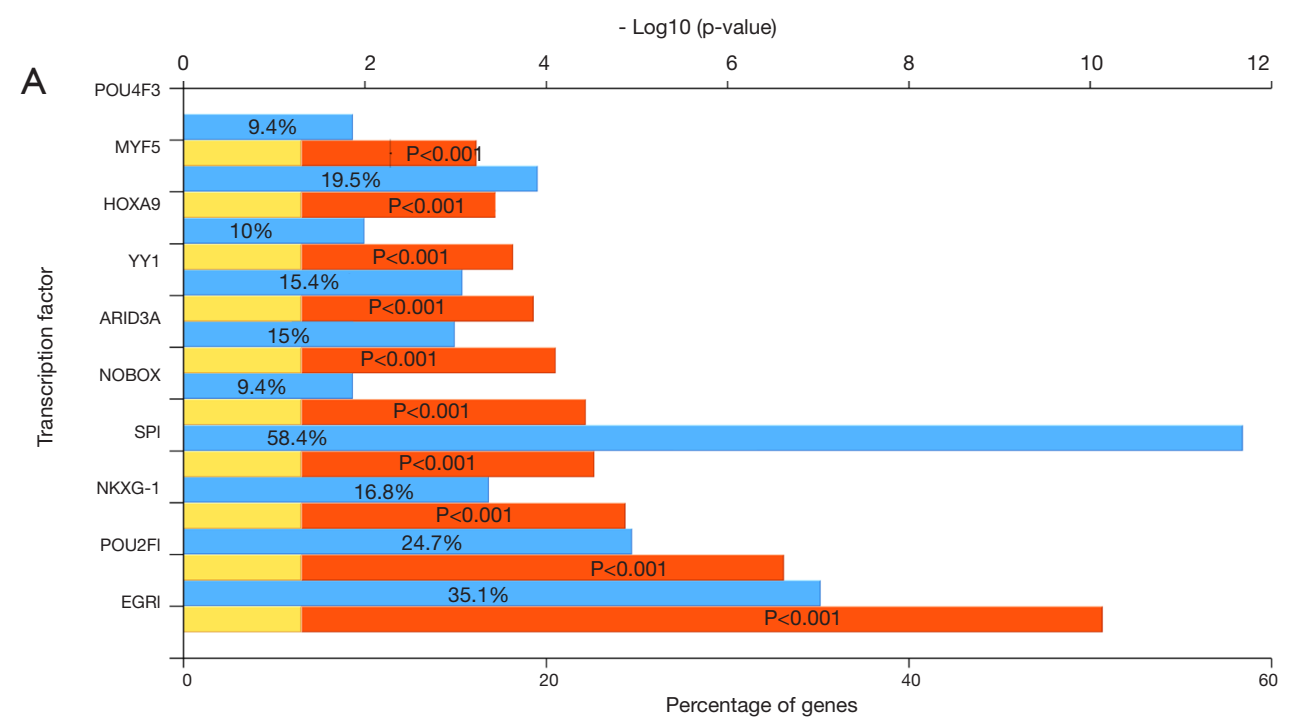

B

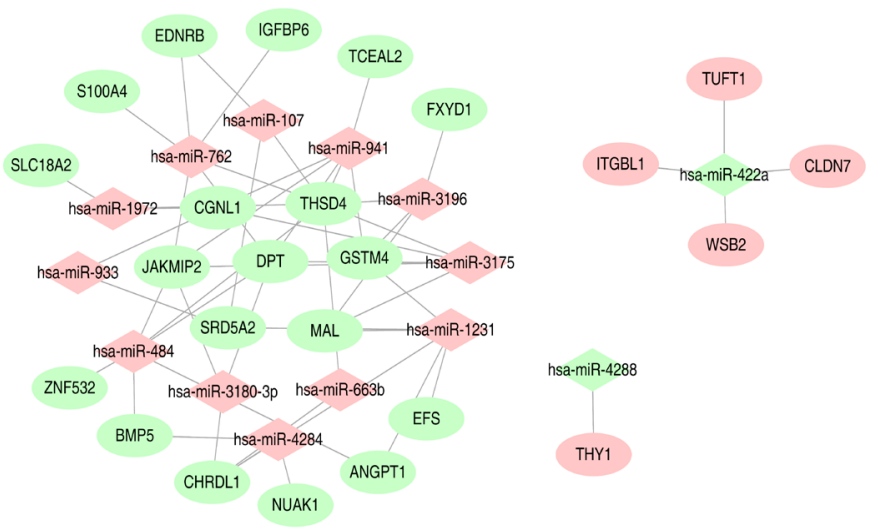

Figure 3 DEmiRNA-DEmRNA interaction network. (A) Top 10 upstream transcription factors of DEmiRNAs. The X-axis shows percentage of genes and the Y-axis represents transcription factors enriched for DEmiRNAs. (B) DEmiRNA-DEmRNA interaction network. The ellipses and rhombuses represent DEmRNAs and DEmiRNAs between PCa and non-tumor control, respectively. Red and green color indicates up- and down-regulation, respectively. DEmRNAs, differentially expressed mRNAs; DEmiRNAs, differentially expressed miRNAs; $\mathrm{PCa}$, prostate cancer. 

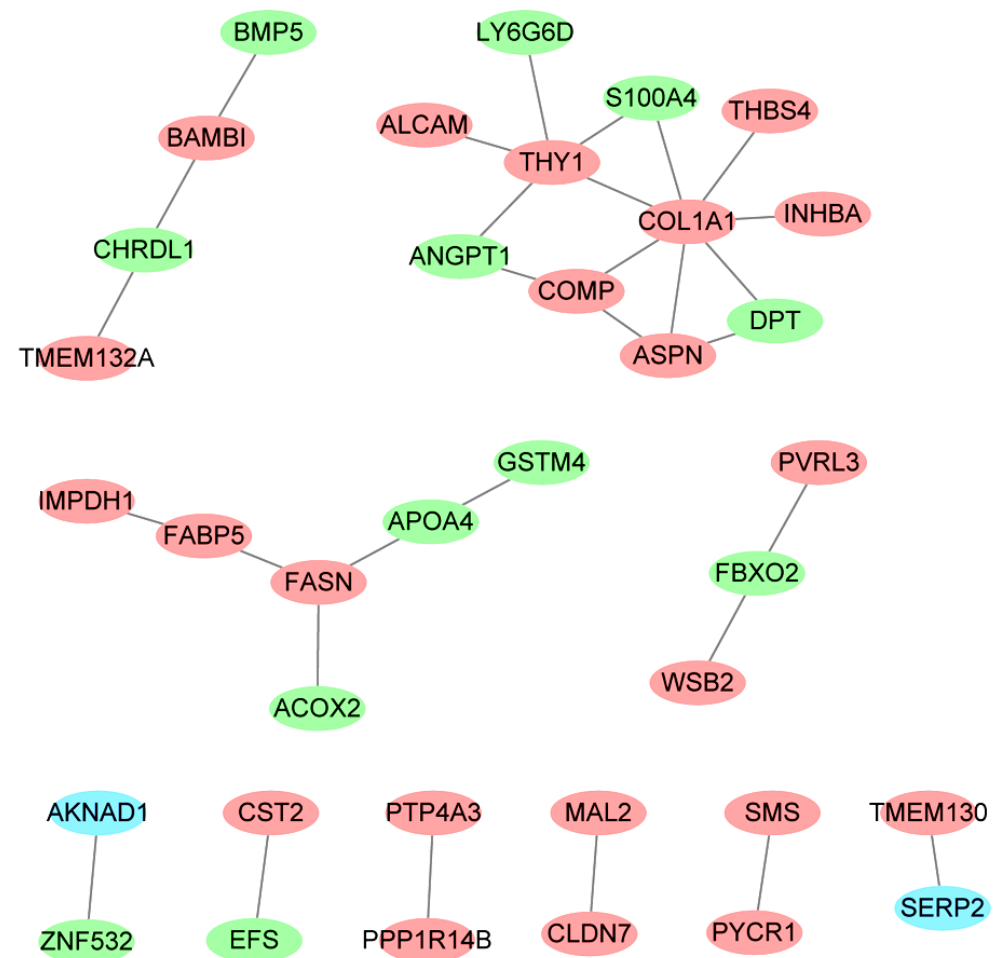

Figure 4 PPI network. Red and green ellipses represent proteins encoded by up- and down-regulated DEmRNAs, respectively. Edges indicate integrations between proteins. Blue ellipses represent other proteins. PPI, protein-protein interaction; DEmRNAs, differentially expressed mRNAs.
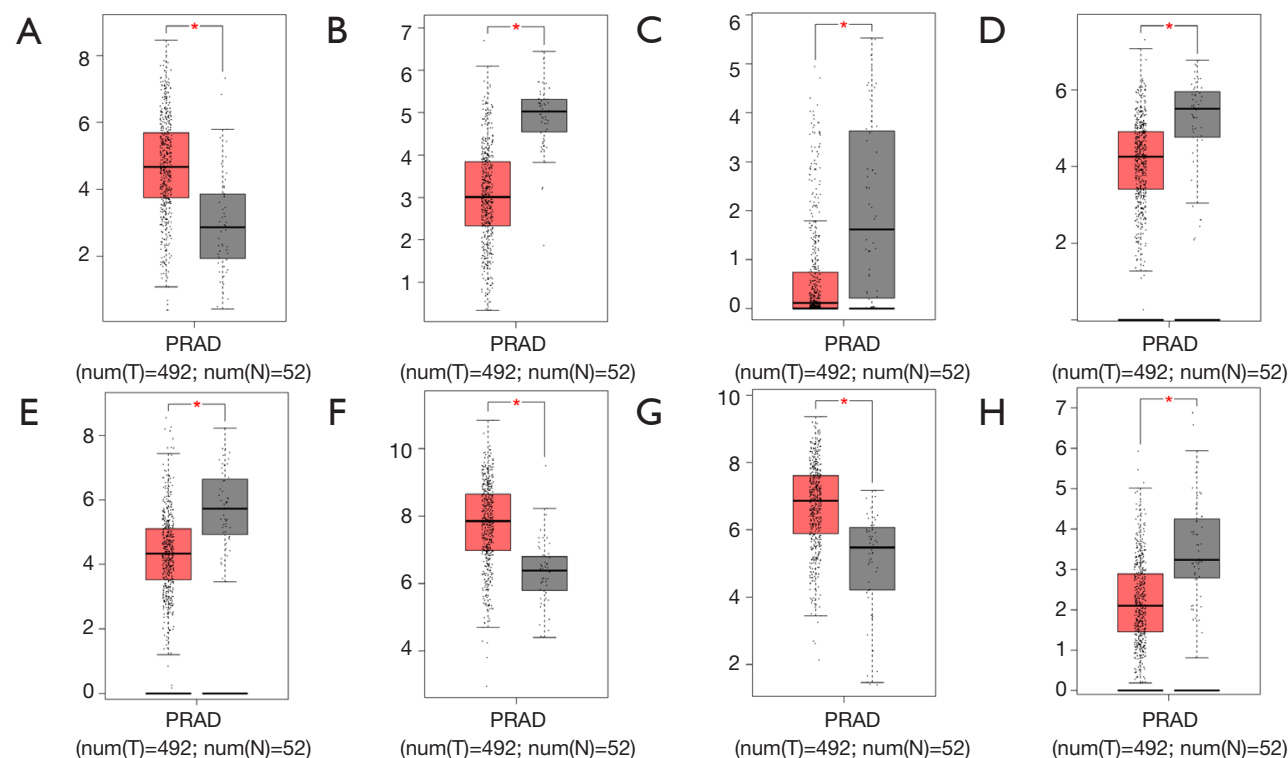

Figure 5 Validation of the expression levels of selected DEmRNAs in PCa based on TCGA database. (A) THBS4; (B) ID4; (C) BMP5; (D) THSD4; (E) FXYD1; (F) PDLIM5; (G) ABCC4; (H) EDNRB. The x-axis shows PRAD group (T) and adjacent non-tumor group (N) and y-axis shows the relative expression levels of DEmRNAs. * indicates $\mathrm{P}$ value $<0.05$. DEmRNAs, differentially expressed mRNAs; PCa, prostate cancer; TCGA, The Cancer Genome Atlas. 

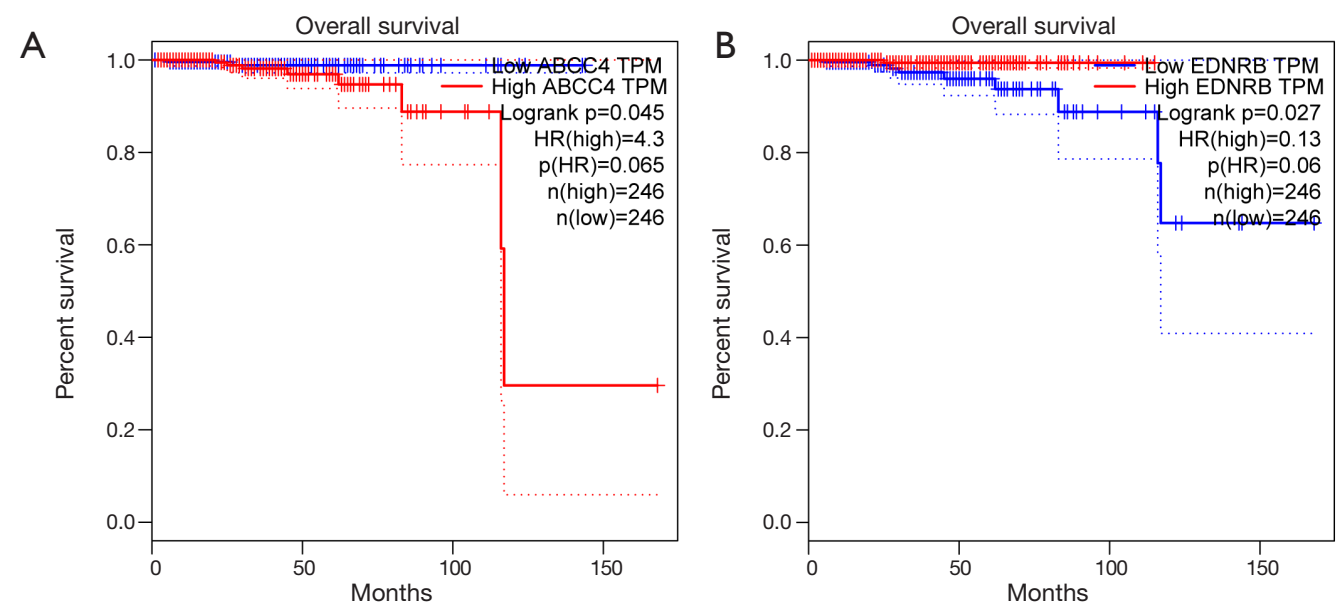

Figure 6 Survival analysis of ABCC4 and EDNRB in PCa. The $\mathrm{x}$-axis shows times (months) and y-axis shows the survival rate. (A) ABCC4; (B) EDNRB. PCa, prostate cancer.

underlying mechanism of $\mathrm{PCa}$ and further experiment was needed to confirm our conclusion.

\section{Acknowledgments}

Funding: This study was supported by Science and Technology Foundation of Tianjin Health Bureau (2015KZ029), the science grant of Tianjin Medical University (2016KYZM05), National Natural Science Foundation of China (81300346), National Natural Science Foundation of China (81702534) and Tianjin Municipal Science and Technology Plan (18ZXDBSY00020).

\section{Footnote}

Conflicts of Interest: All authors have completed the ICMJE uniform disclosure form (available at http://dx.doi. org/10.21037/tcr.2019.12.30). The authors have no conflicts of interest to declare.

Ethical Statement: The authors are accountable for all aspects of the work in ensuring that questions related to the accuracy or integrity of any part of the work are appropriately investigated and resolved. This study has been approved by the ethics institute of The Second Hospital of Tianjin medical university (KY2014K016). All these participants signed the informed consent. This research complied with the principles of the Declaration of Helsinki (as revised in 2013).
Open Access Statement: This is an Open Access article distributed in accordance with the Creative Commons Attribution-NonCommercial-NoDerivs 4.0 International License (CC BY-NC-ND 4.0), which permits the noncommercial replication and distribution of the article with the strict proviso that no changes or edits are made and the original work is properly cited (including links to both the formal publication through the relevant DOI and the license). See: https://creativecommons.org/licenses/by-nc-nd/4.0/.

\section{References}

1. Bray F, Ferlay J, Soerjomataram I, et al. Global cancer statistics 2018: GLOBOCAN estimates of incidence and mortality worldwide for 36 cancers in 185 countries. CA 2018;68:394-424.

2. Center MM, Jemal A, Lortet-Tieulent J, et al. International variation in prostate cancer incidence and mortality rates. Eur Urol 2012;61:1079-92.

3. Kido T, Li Y, Tanaka Y, et al. The X-linked tumor suppressor TSPX downregulates cancer-drivers/oncogenes in prostate cancer in a C-terminal acidic domain dependent manner. Oncotarget 2019;10:1491-506.

4. Liu J, Cheng G, Yang H, et al. Reciprocal regulation of long noncoding RNAs THBS4003 and THBS4 control migration and invasion in prostate cancer cell lines. Mol Med Rep 2016;14:1451-8.

5. Zemskova MY, Song JH, Cen B, et al. Regulation of prostate stromal fibroblasts by the PIM1 protein kinase. 
Cell Signal 2015;27:135-46.

6. Lujambio A, Lowe SW The microcosmos of cancer. Nature 2012;482:347-55.

7. Bartel DP MicroRNAs: target recognition and regulatory functions. Cell 2009;136:215-33.

8. Ivanovic RF, Viana NI, Morais DR, et al. miR-29b enhances prostate cancer cell invasion independently of MMP-2 expression. Cancer Cell Int 2018;18:18.

9. Lin HM, Nikolic I, Yang J, et al. MicroRNAs as potential therapeutics to enhance chemosensitivity in advanced prostate cancer. Sci Rep 2018;8:7820.

10. Lekchnov EA, Amelina EV, Bryzgunova OE, et al. Searching for the Novel Specific Predictors of Prostate Cancer in Urine: The Analysis of 84 miRNA Expression. Int J Mol Sci 2018. doi: 10.3390/ijms19124088.

11. Liu M, Pan H, Zhang F, et al. Identification of TNM stage-specific genes in lung adenocarcinoma by genomewide expression profiling. Oncol Lett 2013;6:763-8.

12. Yang YH, Dudoit S, Luu P, et al. Normalization for cDNA microarray data: a robust composite method addressing single and multiple slide systematic variation. Nucleic Acids Res 2002;30:e15.

13. Diboun I, Wernisch L, Orengo CA, et al. Microarray analysis after RNA amplification can detect pronounced differences in gene expression using limma. BMC Genomics 2006;7:252.

14. Pathan M, Keerthikumar S, Chisanga D, et al. A novel community driven software for functional enrichment analysis of extracellular vesicles data. J Extracell Vesicles 2017;6:1321455.

15. Tang Z, Li C, Kang B, et al. GEPIA: a web server for cancer and normal gene expression profiling and interactive analyses. Nucleic Acids Res 2017;45:W98-W102.

16. McCart Reed AE, Song S, Kutasovic JR, et al.

Thrombospondin-4 expression is activated during the stromal response to invasive breast cancer. Virchows Arch 2013;463: 535-45.

17. Greco SA, Chia J, Inglis KJ, et al. Thrombospondin-4 is a putative tumour-suppressor gene in colorectal cancer that exhibits age-related methylation. BMC Cancer 2010;10:494.

18. Förster S, Gretschel S, Jons T, et al. THBS4, a novel stromal molecule of diffuse-type gastric adenocarcinomas, identified by transcriptome-wide expression profiling. Mod Pathol 2011;24:1390-403.

19. Dakhova O, Ozen M, Creighton CJ, et al. Global gene expression analysis of reactive stroma in prostate cancer. Clin Cancer Res 2009;15:3979-89.
20. Vasiljević N, Wu K, Brentnall AR, et al. Absolute quantitation of DNA methylation of 28 candidate genes in prostate cancer using pyrosequencing. Dis Markers 2011;30:151-61.

21. Phé V, Cussenot O, Roupret M Methylated genes as potential biomarkers in prostate cancer. BJU Int 2010;105: 1364-70.

22. Bova GS, Kallio HM, Annala M, et al. Integrated clinical, whole-genome, and transcriptome analysis of multisampled lethal metastatic prostate cancer. Cold Spring Harb Mol Case Stud 2016;2:a000752.

23. Demidenko R, Razanauskas D, Daniunaite K, et al. Frequent down-regulation of $\mathrm{ABC}$ transporter genes in prostate cancer. BMC Cancer 2015;15:683.

24. Wang WX, Kyprianou N, Wang X, et al. Dysregulation of the mitogen granulin in human cancer through the miR-15/107 microRNA gene group. Cancer Res 2010;70:9137-42.

25. Katar S, Baran O, Evran S, et al. Expression of miRNA-21, miRNA-107, miRNA-137 and miRNA-29b in meningioma. Clin Neurol Neurosurg 2017;156:66-70.

26. Liu F, Liu S, Ai F, et al. miR-107 Promotes Proliferation and Inhibits Apoptosis of Colon Cancer Cells by Targeting Prostate Apoptosis Response-4 (Par4). Oncol Res 2017;25:967-74.

27. Zhou Q, Zheng X, Chen L, et al. Smad2/3/4 Pathway Contributes to TGF-beta-Induced MiRNA-181b Expression to Promote Gastric Cancer Metastasis by Targeting Timp3. Cell Physiol Biochem 2016;39:453-66.

28. Zimmerman KA, Xing D, Pallero MA, et al. Calreticulin Regulates Neointima Formation and Collagen Deposition following Carotid Artery Ligation. J Vasc Res 2015;52:306-20.

29. Ren X, Bo Y, Fan J, et al. Dalbergioidin Ameliorates Doxorubicin-Induced Renal Fibrosis by Suppressing the TGF-beta Signal Pathway. Mediators Inflamm 2016;2016:5147571.

30. Tennant BR, Chen J, Shih AZ, et al. Myt3 Mediates Laminin-V/Integrin-beta1-Induced Islet-Cell Migration via Tgfbi. Mol Endocrinol 2015;29:1254-68.

31. Zhao J, Cheng Q, Ye P, et al. Atorvastatin improves pathological changes in the aged kidney by upregulating peroxisome proliferator-activated receptor expression and reducing matrix metalloproteinase-9 and transforming growth factor-beta1 levels. Exp Gerontol 2016;74:37-42.

32. Bentley H, Hamdy FC, Hart KA, et al. Expression of bone morphogenetic proteins in human prostatic adenocarcinoma and benign prostatic hyperplasia. Br J 
Cancer 1992;66:1159-63.

33. Wordinger RJ, Agarwal R, Talati M, et al. Expression of bone morphogenetic proteins (BMP), BMP receptors, and BMP associated proteins in human trabecular meshwork and optic nerve head cells and tissues. Mol Vis 2002;8:241-50.

34. Li Y, Shen Z, Jiang H, et al. MicroRNA4284 promotes gastric cancer tumorigenicity by targeting ten-eleven translocation 1. Mol Med Rep 2018;17:6569-75.

35. Zare A, Ahadi A, Larki P, et al. The clinical significance of miR-335, miR-124, miR-218 and miR-484 downregulation in gastric cancer. Mol Biol Rep 2018;45:1587-95.

36. Lu X, Lu J The significance of detection of serum miR423-5p and miR-484 for diagnosis of colorectal cancer. Clin Lab 2015;61:187-90.

37. Su P, Wen S, Zhang Y, et al. Identification of the Key Genes and Pathways in Esophageal Carcinoma. Gastroenterol Res Pract 2016;2016:2968106.

Cite this article as: $\mathrm{Wu} \mathrm{J}, \mathrm{Li} \mathrm{X}$, Luo F, Yan J, Yang K. Screening key miRNAs and genes in prostate cancer by microarray analysis. Transl Cancer Res 2020;9(2):856-868. doi: 10.21037/tcr.2019.12.30
38. Cohen H, Ben-Hamo R, Gidoni M, et al. Shift in GATA3 functions, and GATA3 mutations, control progression and clinical presentation in breast cancer. Breast Cancer Res 2014;16:464.

39. Ma J, Hou X, Li M, et al. Genome-wide methylation profiling reveals new biomarkers for prognosis prediction of glioblastoma. J Cancer Res Ther 2015;11 Suppl 2:C212-5.

40. Fomicheva KA, Osip'yants AI, Knyazev EN, et al. Detection of Potential Metastatic Prostate Cancer Circulating Biomarkers by Comparison of miRNA Profiles in DU145 Cells and Culture Medium. Bull Exp Biol Med 2017;162:792-6.

41. Guo X, Han T, Hu P, et al. Five microRNAs in serum as potential biomarkers for prostate cancer risk assessment and therapeutic intervention. Int Urol Nephrol 2018;50:2193-200. 